\title{
REAL-TIME MONITORING OF COSMIC RAY ANISOTROPY PARAMETERS AND SHORT-TERM FORECASTING OF GEOMAGNETIC DISTURBANCES
}

\author{
A.S. Zverev \\ Yu.G. Shafer Institute of Cosmophysical Research \\ and Aeronomy $S B R A S$, \\ Yakutsk, Russia,zverevas@ikfia.ysn.ru \\ V.G. Grigoryev \\ Yu.G. Shafer Institute of Cosmophysical Research \\ and Aeronomy SB RAS, \\ Yakutsk,Russia,grig@ikfia.ysn.ru
}

\author{
P.Yu. Gololobov \\ Yu.G. Shafer Institute of Cosmophysical Research \\ and Aeronomy SB RAS, \\ Yakutsk,Russia, gpeter@ikfia.ysn.ru \\ S.A. Starodubtsev \\ Yu.G. Shafer Institute of Cosmophysical Research \\ and Aeronomy $S B R A S$, \\ Yakutsk, Russia,starodub@ikfia.ysn.ru
}

\begin{abstract}
SHICRA SB RAS, using data from the NMDB database, has implemented continuous monitoring of the dynamics of cosmic ray (CR) distribution parameters and the automatic forecasting of geomagnetic disturbance. Monitoring is based on the global survey method, which considers the world-wide network of neutron monitors as a single device oriented in different directions at each measured time. This method provides real-time parameters of nine components of the first two angular moments of the CR distribution function for each hour of observation. This paper discusses methodological aspects related to the use of the global survey
\end{abstract}

method and some results of the forecasting of geomagnetic disturbances for 2017-2018.

Keywords: cosmic rays, neutron monitor, global survey, geomagnetic storms, zonal components, predictors.

\section{INTRODUCTION}

The topical area of research on variations of the cosmic ray (CR) intensity is forecasting of geoeffective disturbances of the solar wind (SW). Earlier studies of $\mathrm{CR}$ rigidity spectrum variations carried out by different authors [Dvornikov et al., 1988, 1995; Belov et al., 2001; Dorman et al., 2003; Munakata et al., 2005; Grigoryev et al., 2006] have shown the fundamental possibility for their forecasting based on geoeffective SW disturbances. Currently, there are various methods for realtime forecasting of interplanetary medium disturbances through the analysis of CR anisotropy, for example [http://neutronm.bartol.udel.edu/spaceweather]. SHICRA SB RAS implements the monitoring using a global survey method, with the world-wide network of CR stations, equipped with neutron monitors, considered as a single multipurpose device. The CR intensity observed by each detector is generally determined by receiving characteristics of a detector, which represent its geometry, geographical location, and type of secondary particles observed, and are joined by the concept of receiving vector. Note that the global survey method was developed by SHICRA SB RAS in the late 1960s to study CR distribution in interplanetary space [Krymsky et al., 1981].

The creation and development of the international neutron monitor database NMDB [http://www01.nmdb. eu] opened up the possibility for using the global survey method in real time [Grigoryev, Starodubtsev, 2015]. This eventually led to the development of a method for real-time short-term (from several hours to $\sim 1$ day) forecasting of geomagnetic storms with the Dst index less than $-50 \mathrm{nT}$. Forecasting results are available at [http://www.ysn.ru/ starodub/SpaceWeather/global_sur vey_real_time.html]

\section{EXPERIMENTAL DATA AND MONITORING METHOD}

Using data from the world-wide network of neutron monitors in real time requires its preprocessing. In this case, we take on risk of using raw data. Information from the neutron monitor stations is stored in DB in real time, and its use in this mode requires us to solve a number of methodological problems. Besides the usual, quite recoverable short-term hardware failures there are changes in the level of count, linear trend, and other effects. A detailed presentation of all the failures observed in CR data and the problems to be solved before using the data is quite extensive, but we briefly discuss some of them, describing at the same time how to fix the failures. The short-term hardware failures are removed on the basis of Chauvenet's criterion [Taylor, 1985]. One of the main problems of data preparation is to correctly identify lowand high-frequency parts of the CR intensity variations the device recorded. In this case, the high-frequency part of CR intensity variations at each station for the 24-hour interval is identified as the difference between the observed variations and the trend. Since it is known [Otnes, Enochson, 1982] that the data filtering procedure does not completely remove linear trends, to eliminate them we use the standard least-squares method. Moreover, the experiment demonstrates the dependence of the required CR distribution parameters on the solar activity level, which should be taken into account using coupling coefficients for neutron monitors. 
As shown by our studies, the main predictors in $\mathrm{CR}$ that actively respond to geoeffective disturbances of the interplanetary medium approaching Earth are changes in amplitudes of zonal (north-south) components of the high-frequency part of the isotropic CR intensity $\left(C_{00}\right)$ and the first two moments of the angular distribution $\left(C_{10}\right.$ and $\left.C_{20}\right)$ [Grigoryev et al., 2017], which are included in the nine parameters determined by the global survey method [Krymsky et al., 1981] and may take both positive and negative values with respect to the ecliptic plane. The predictors may also be changes in the value and direction of the first harmonic vector and in the sum of amplitudes of zonal components, which occur before the interplanetary disturbance arrives in Earth's orbit. We can empirically determine the critical levels above which positive values of any of the three components or their sums most likely predict an abrupt decrease in the Dst index at the beginning of a geomagnetic storm [Grigoryev et al., 2019]. Yet, a significant decrease in negative values of these components or their sums below a certain critical value may indicate the most disturbed phase of the geomagnetic storm.

The dynamics of the main CR distribution parameters for the last three days: isotropic intensity $I$, harmonic vector modulus $\overrightarrow{A_{1}^{1}}$, and sums of positive and negative values of zonal harmonics, as well as components and vectors of the first $\overrightarrow{A_{1}^{1}}$ and second $\overrightarrow{A_{2}^{2}}$ harmonics in the GSE coordinate system is shown at [http://www.ysn.ru/ starodub/SpaceWeather/global_survey_real_time.html].

\section{RESULTS}

In recent years, against low solar activity there is very low geomagnetic activity. Nevertheless, over the period 2017-2019 there were four significant magnetic storms that occurred on May 28 (Dst $\approx-125 \mathrm{nT}$ ), July $16(D s t \approx-72 \mathrm{nT})$, and September $7(D s t \approx-124 \mathrm{nT})$, 2017, and on August 25, 2018 (Dst $\approx-174$ nT) [http://wdc.kugi.kyoto-u.ac.jp]. As an example, Figure presents the sums of positive $\sum C^{+}=C^{+}{ }_{00}+C^{+}{ }_{10}+C^{+}{ }_{20}$ and negative $\sum C^{-}=C_{00}^{-}+C_{10}{ }_{10}+C_{20}^{-}$zonal $\mathrm{CR}$ anisotropy components, as well as Dst variations during these geomagnetic storms. The sums of positive values of zonal components above 0.9 and negative ones below -1.2 suggest that they reach or exceed critical values, which we interpret as moments of occurrence of predictors of the onset and the main phase of a geomagnetic storm respectively. Figure demonstrates that for these geomagnetic storms predictors occur two hours to one day before the onset of the geomagnetic disturbance.

\section{CONCLUSIONS}

1. We have analyzed four severest magnetic storms that occurred in 2017-2018. All of them had predictors in CR distribution components obtained by the global survey method.

2. The global survey method used for determining CR anisotropy parameters enables an automatic shortterm (from few hours to $\sim 1$ day) forecasting of geomagnetic storms with $D s t \leq-50 \mathrm{nT}$.
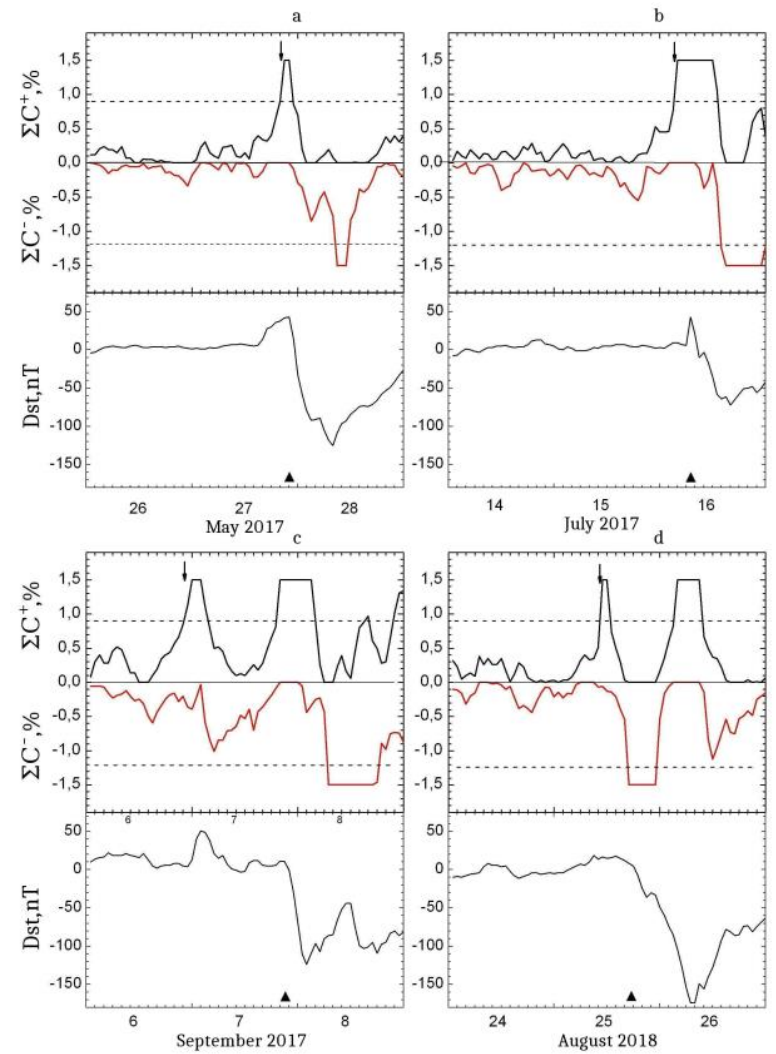

Time dependence of sums of positive $\left(\Sigma C^{+}\right)$and negative $\left(\Sigma C^{-}\right)$zonal harmonics, as well as Dst variations during magnetic storms on May $28(a)$, July $16(b)$, September 7, 2017 (c), and August 25, 2018 (d). Dashed lines indicate critical levels for predictors of geomagnetic storms; triangles mark onsets of storms; arrows, beginning of occurrence of the predictors

3. It is necessary to further improve the monitoring, including direct measurements of interplanetary medium parameters.

The work was performed using the Unique Research Facility Russian National Ground-Based Network of Cosmic Ray Station [http://ckp-rf.ru/usu/433536] with support from RFBR grant No. 18-42-140002-r_a. We acknowledge the NMDB database (www.nmdb.eu), founded under the European Union's FP7 programme (contract no. 213007), for providing data.

\section{REFERENCES}

Belov A.V., Bieber J.W., Eroshenko E.A., Evenson P. Pitch-angle features in cosmic rays in advance of severe magnetic storms: neutron monitor observations. Proc. $27^{\text {th }}$ International Cosmic Ray Conference. Hamburg, 2001, vol. 9, pp. 3507-3510.

Dorman L.I., Belov A.V., Eroshenko E.A., Pustil'nik L.A., Sternlieb A., Yanke V.G., Zukerman I.G. Possible cosmic ray using for forecasting of major geomagnetic storms, accompanied by Forbush effects. Proc. $28^{\text {th }}$ International Cosmic Ray Conference. Tsukuba, 2003, vol. 6, pp. 3553-3556.

Dvornikov V.M., Sdobnov V.E., Sergeev A.V. Method for prediction of sporadic geoeffective perturbations of solar wind: Patent RU 1769602 C. 1995.

Dvornikov V.M., Sergeev A.V., Sdobnov V.E. Abnormal variations of cosmic rays in rigidity range $2-5 \mathrm{GV}$ and their relation ti heliospheric disturbances. Izvestiya Akademii Nauk 
SSSR. Seriya fizicheskaya [Bulletin of the Academy of Sciences of USSR: Physics]. 1988, vol. 52, no. 12, pp. 2435-2437. (In Russian).

Grigoryev V.G., Starodubtsev S.A. Global survey method in real time and space weather forecasting. Bulletin of the Russian Academy of Sciences: Physics. 2015, vol. 79, no. 5, pp. 649-653. DOI: 10.3103/S1062873815050226.

Grigoryev V.G., Starodubtsev S.A., Gololobov P.Y. Monitoring geomagnetic disturbance predictors using data of ground measurements of cosmic rays. Bulletin of the Russian Academy of Sciences: Physics. 2017, vol. 81, no. 2, pp. 200202. DOI: $10.3103 / \mathrm{S} 1062873817020198$.

Grigoryev V.G., Starodubtsev S.A., Gololobov P.Yu. Monitoring of geomagnetic disturbances using the global survey method in real time. Solar-Terrestrial Physics. 2019, vol. 5 , iss. 3, pp. 93-97. DOI: 10.12737/stp-53201911.

Grigoryev V.G., Starodutbsev S.A., Krivoshapkin P.A., Prihod'ko A.N., Egorov A.G., Turpanov A.A. Cosmic ray anisotropy according to Yakutsk station data in real time. Trudy Vserossiiskoi konferetsii "Eksperimental'nye $i$ teoreticheskie issledovaniya osnov prognozirovaniya geliogeofizicheskoi aktivnosti" [Proceedings of All-Russian Conference "Experimental and Theoretical Investigation on the Basics of Forecasting of Heliogeophysical Activity". October 10-15, 2005, Troitsk, IZMIRAN]. 2006, pp. 79-84. (In Russian).

Krymsky G.F., Kuzmin A.I., Krivoshapkin P.A., Samsonov I.S., Skripin G.V., Transky I.A., Chirkov N.P. Kosmicheskie luchi $i$ solnechnyi veter [Cosmic Rays and Solar Wind]. Novosibirsk, Nauka Publ., 1981, 224 p. (In Russian).

Munakata K., Kuwabara T., Yasue S., Kato C., Akahane S., Koyama M. A "loss cone" precursor of an approaching shock observed by a cos-mic ray muon hodoscope on October 28, 2003. Geophys. Res. Lett. 2005, vol. 32, L03S04. DOI: 1029/2004GL021469.
Otnes R., Enochson L. Prikladnoi analiz vremennykh ryadov. Osnovnye metody [Applied analysis of time series. Basic methods]. Moscow, Mir Publ., 1982, 432 p. (In Russian). (English edition: Otnes R., Enochson L. Applied Time Series Analysis: Basic Techniques. Wiley, 1978, 449 p.)

Teilor Dzh. Vvedenie v teoriyu oshibok [Introduction to error analysis]. Moscow, Mir Publ., 1985, 272 p. (In Russian). (English edition: Taylor J. An Introduction to Error Analysis: The Study of Uncertainties in Physical Measurements. University Science Books, 1982, 270 p.)

URL: http://neutronm.bartol.udel.edu/spaceweathe (accessed June 15, 2020).

URL: http://www01.nmdb.eu (accessed June 15, 2020).

URL:

http://www.ysn.ru/ starodub/SpaceWeather/global_survey_re al time.html (accessed June 15, 2020).

URL: http://wdc.kugi.kyoto-u.ac.jp (accessed June 15, 2020).

URL: http://ckp-rf.ru/usu/433536 (accessed June 15, 2020).

How to cite this article

Zverev A.S., Grigoryev V.G., Gololobov P.Yu., Starodubtsev S.A. Real-time monitoring of cosmic ray anisotropy parameters and short-term forecasting of geomagnetic disturbances. Solar-Terrestrial Physics. 2020. Vol. 6. Iss. 4. P. 37-39. DOI: 10.12737/stp-64202005. 